\title{
Stabilité des pentes sous-marines dans la Baie des Anges, Nice, France. Approche géotechnique.
}

\section{Offshore slope stability in the Baie des Anges, Nice, France. Geotechnical approach.}

\author{
Th. MULDER*, J.-P. TISOT* \\ P. COCHONAT ${ }^{* *}$, J.-F. BOURILLET**
}

Rev. Franç. Géotech. n 64, pp. 21-30 (juillet 1993)

\section{Résumé}

La Baie des Anges présente des singularités qui en font un site exemplaire pour l'étude des instabilités sous-marines. L'application du modèle de glissement plan en tenant compte de l'activité sismique a permis de mettre en évidence l'instabilité potentielle d'une part importante de la baie. Deux types de glissements sont possibles: de petits réajustements locaux de la pente impliquant des sédiments sous-consolidés du haut du plateau continental et de grands glissements de flancs de crêtes, dont le volume peut permettre la transformation en courants de turbidité.

\footnotetext{
Abstract

The Baie des Anges shows some particularities. Consequently, it is a model site for the offshore instabilities study. The application of the infinite slope analysis including the seismicity, allows to prove the potential instability of the area. Two kinds of slides are possibles : firstly, local readjustments of the slope, involving the top-continental plate underconsolidated sediments; secondly, huge ridge-side instabilities. Their volume allows the transformation of the slides into some turbidity currents.
}

\footnotetext{
- Laboratoire de Géomécanique, ENSG, rue du doyen Marcel-Roubault, BP 40, 54501 Vandøeuvre-lès-Nancy.

** IFREMER-GM, Centre de Brest, BP 70, 29280 Plouzané.
} 


\section{CARACTÉRISTIQUES MORPHOLOGIQUES, GÉOLOGIQUES ET GÉOTECHNIQUES DE LA BAIE DES ANGES}

La Baie des Anges se caractérise par la présence d'un plateau continental réduit. La couverture sédimentaire se termine par $1000 \mathrm{~m}$ de sédiments plio-quaternaires surmontant la surface d'érosion messinienne (SAVOYE and al., 1989; SAVOYE and PIPER, 1991). Le talus continental présente de fortes valeurs du gradient de pente accentuées par la présence de deux canyons entaillant la baie: les canyons du Var et du Paillon (fig. 1). Localement, les valeurs du gradient de pente peuvent dépasser $60 \%$ (fig. 2). Ainsí, après un dépôt rapide, les silts argileux superficiels sont soumis actuellement à de

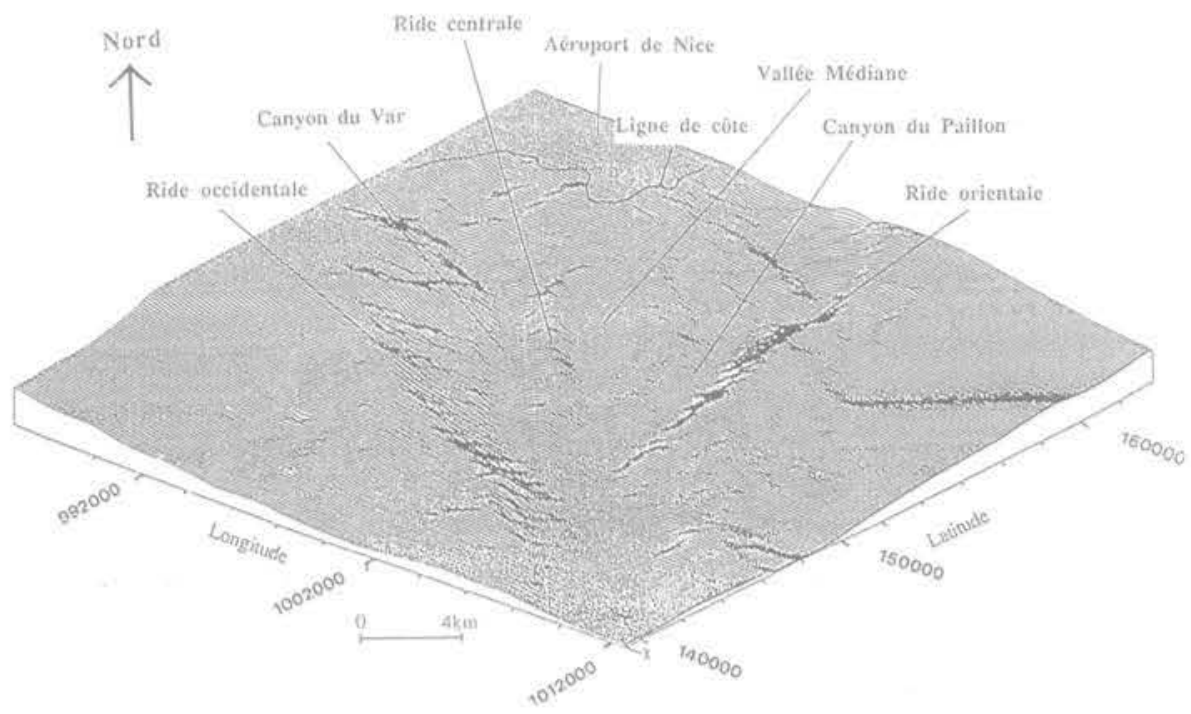

Fig. 1. - Bloc diagramme montrant la morphologie générale de la baie.

Fig. 1. - 3D diagram showing the bottom morphology of the bay.

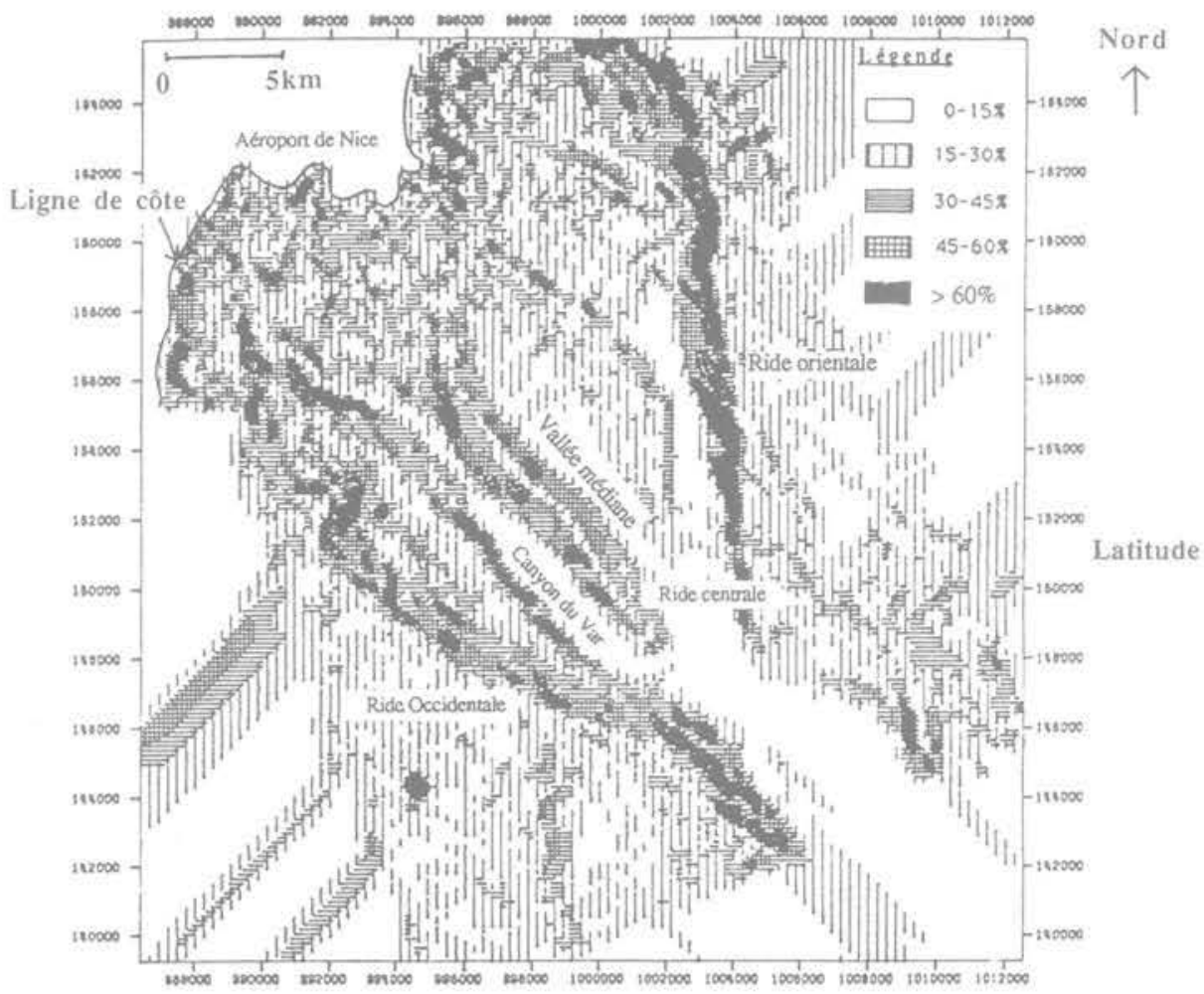

Longitude

Fig. 2. - Carte du gradient de pente réelle dans la Baie des Anges (données IFREMER, d'après BOURILLET, 1992).

Fig. 2. - Slope gradient map in the Baie des Anges (IFREMER Data, after BOURILLET, 1992). 
nombreux glissements en masse (COCHONAT et al., 1988 ; SAVOYE and al., 1989; SCHIEB et al., 1990), qui correspondent à des réajustements locaux de la morphologie. Ces glissements peuvent affecter les installations humaines, comme ce fut le cas en 1979 au chantier de l'aéroport de Nice. Il s'avère donc nécessaire, si ce n'est de les prévoir, du moins, de localiser les zones potentielles de départ de tels glissements.

Outre un risque d'instabilités statiques, la Baie des Anges peut aussi être l'objet d'instabilités dynamiques: en plus d'une importante sismicité interne, la baie subit l'influence de séismes plus fréquents et de plus fortes magnitudes dont les épicentres se situent en mer Ligure (REHAULT et BETHOUX, 1984) ou sur la partie continentale de la chaîne alpine.

\section{MORPHOLOGIE DES GLISSEMENTS SOUS-MARINS}

Les glissements sous-marins ont été étudiés en détail dans le delta du Mississippi. Ce dernier présente une palette quasiment complète des différents types d'instabilités (COLEMAN and GARRISON, 1977; COLE. MAN and PRIOR, 1981; PRIOR and COLEMAN, 1982).

Certaines de ces instabilités sont directement liées à l'activité tectonique. Elles prennent alors le plus souvent la forme de remplissage d'escarpements créés lors d'épisodes distensifs (génération de fossés d'effondrement), ou lors d'une activité tectonique synsédimentaire. Les instabilités sont alors liées à des glissements rotationnels anciens situés sur la partie supérieure du plateau continental, ou aux failles de croissance de la partie sommitale du talus.

La forme de glissement la plus répandue en mer, que ce soit à l'échelle mondiale ou dans le cadre plus res. treint de la Baie des Anges, est le glissement plan qui rassemble des morphologies, des volumes et des natures de matériau très diverses. Sa caractéristique principale est un rapport épaisseur/longueur très faible et inférieur à la valeur limite de 0,15 définie par SKEMPTON et HUTCHINSON (1969). La nature, cohérente ou non, définit le devenir du phénomène gravitaire : glissement en masse ou coulée (liquéfaction possible du matériau). Ce type d'accident peut indirectement être lié à l'activité tectonique, puisque les accélérations sismiques générées par un tremblement de terre peuvent être à l'origine de la mise en mouvement.

En effet, en fonction de l'angle de pente et de la masse de sédiments impliquée dans le glissement, la dynamique de ce dernier peut être considérablement accélérée et "dégénérer * vers un mode de transport en suspension (phénomène d'ignition) pour finalement se transformer en courant de turbidité. Le matériau se dé. pose alors aux endroits où le gradient de pente prend des valeurs très faibles (valeurs inférieures à $1^{\circ}$ ). La distance parcourue est considérable. Le glissement du Grand Bank (Canada) (HEEZEN and EWING, 1952; KUENEN, 1952; CLARK and LANDVA, 1991) a par- couru près de 700 kilomètres depuis son point d'origine. L'ordre de grandeur est légèrement moindre pour les glissements qui peuvent affecter la Baie des Anges. A titre d'exemple, le glissement de 1979 a parcouru environ $130 \mathrm{~km}$ (fig. 3).

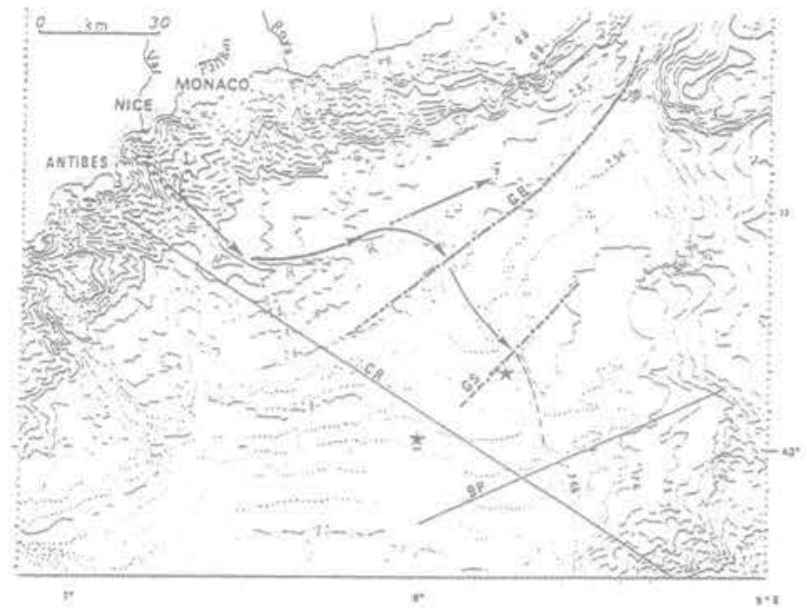

Fig. 3. - Origine du glissement et chemin parcouru par l'événement de 1979 (d'après GENNESSAUX et al., 1980).

Fig. 3. - Origin and travel for the 1979 slump (from GENNESSAUX et al. 1980).

\section{NATURE DES SÉDIMENTS SUPERFICIELS DANS LA BAIE DES ANGES}

Plusieurs missions de l'IFREMER ont permis une connaissance précise des sédiments de la baie: missions Prénice, Same (SCHIEB, 1992), Casanice et Catherine. Laurence (avec la collaboration du Laboratoire de Géodynamique sous-marine de Villefranche-sur-Mer) (MULDER, 1992).

Les sédiments superficiels de la Baie des Anges sont principalement constitués de silts argileux fins, bien classés et riches en carbonates ( 30 à $50 \%$ ). L'utilisation des limites d'Atterberg montre une répartition dans les silts organiques peu plastiques sur le diagramme de Casagrande $\left(7<I_{<}<20\right.$ et $\left.30<W_{<}<45\right)$. Outre les carbonates, les minéraux principaux (déterminés par diffraction RX) sont: le quartz, les plagioclases, les micas et la chlorite. Si cette description résume bien les caractéristiques des sédiments du haut de plateau continental et de ceux du flanc et du sommet des rides et du talus, il est important de noter que les fonds des canyons du Var et du Paillon sont constitués de matériaux plus grossiers, pouvant être assimilés à des coulées de débris. Les principales caractéristiques de ces deux grands types de sédiments sont résumées d'une part d'un point de vue granulométrique (fig. 4) et d'autre part, sur les coupes de la figure 5.

On détaillera ci-dessous l'état des sédiments, mis à part pour ceux situés au fond des canyons. En ce qui concerne la résistance au cisaillement à long terme, elle peut être définie par $c^{\prime}=5 \mathrm{kPa}$ et $\varphi^{\prime}=35^{\circ}$ ). 


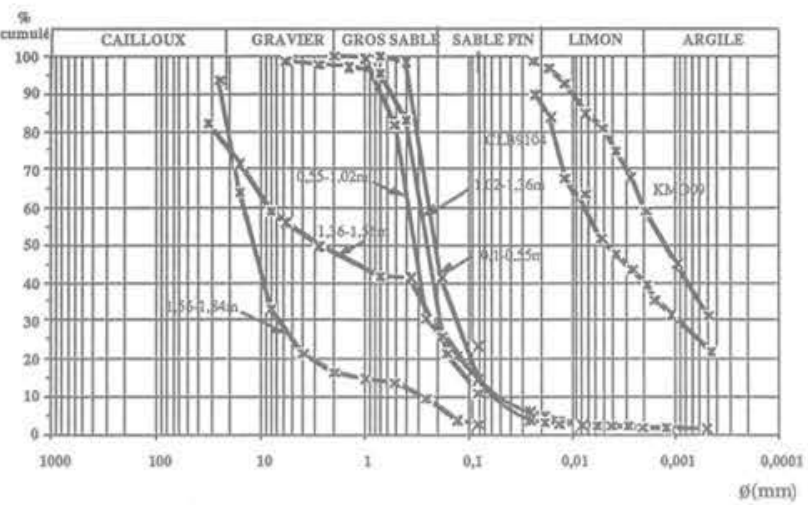

Fig, 4. - Exemples de granulométrie pour les sédiments sur plateau continental, sur le talus et sur les rides (exemples des prélèverments CLB 9104 et KMO 09) et pour les sédiments grossiers du fond de canyons (prélèvement CLK 9101).

Fig. 4. - Typical granulometric curves for the sediments localized on the plate, on the continental slope or on the ridges (exemples of CLB 9104 and KMO 09 cores) and for the coarser sediments of the canyons (CLK 9101 core).

\section{4. ÉTAT DES SÉDIMENTS}

La caractérisation de l'état des sédiments a été réalisée soit au moyen d'essais en place, soit à partir de mesures de laboratoire sur des carottes (SCHIEB, 1992; MUL. DER, 1992).

In situ, deux types d'appareillages ont été utilisés (CO. CHONAT et al., 1990; COCHONAT, 1991b). Il s'agit d'une part du scissomètre grand fond et d'autre part du module géotechnique, composé d'un piézocône et d'un carottier associé. Ces deux outils donnent des résultats de qualité, mais leur profondeur d'investigation est limitée à moins d'un mètre pour le premier et à deux mètres pour le second.

Au laboratoire, les paramètres mesurés pour caractériser l'état du sédiment sont la teneur en eau, le poids volumique $\left(\gamma_{h}\right)$, la cohésion non-drainée (c) (fig. 5) et la pression de préconsolidation $\left(\sigma_{p}^{\prime}\right)$. Les trois premières mesures sont rapides et assez fiables. En revanche, la détermination de la pression de préconsolidation est beaucoup plus longue et nécessite que les remaniements soient très limités. La vérification pour les silts argileux de la baie de la constance du rapport $c_{y} / \sigma^{\prime}$ à partir de quelques couples de valeurs, permet d'utiliser les mesures systématiques de cohésion non-drainée pour évaluer en tout point, la valeur de la pression de préconsolidation.

Globalement, on observe pour l'ensemble des sédiments de la Baie des Anges, l'existence d'une surconsolidation de surface de type quasi-surconsolidation (TISOT, 1986), liée à des phénomènes physico-chimiques.

Une analyse plus précise de l'état de consolidation a permis la séparation des sédiments en quatre grandes familles géotechniques (COCHONAT et al., 1992), à chacune desquelles a pu être associée une loi de varia- tion de la cohésion non-drainée c, exprimée en $\mathrm{kPa}$, avec la profondeur $z$, en mètres (fig. 6).

- Zone A (sédiments sous-consolidés): $c_{u}=1,35 . z$ $(\mathrm{kPa})$;

- Zone B (sédiments normalement consolidés) :

$c_{\mathrm{u}}=1,2.2+5(\mathrm{kPa})$;

- Zone $\mathrm{C}$ (sédiments légèrement surconsolidés) :

$c_{u}=2,5 . z+5(\mathrm{kPa})$;

- Zone D (sédiments surconsolidés) : $c_{u}=2,5 . z+15$ $(\mathrm{kPa})$.

Il existe une corrélation entre l'état de consolidation des matériaux et leur répartition à travers les grands ensembles géomorphologiques de la baie: les sédiments sous-consolidés sont en majeure partie localisés sur la partie sommitale du plateau continental, les sédiments surconsolidés sur les sommets, les flancs de crêtes, le talus étant en règle générale constitués superficiellement de sédiments normalement consolidés. Cette corrélation a permis d'extrapoler les données ponctuelles de carottages afin de définir une zonation géotechnique de la baie (fig. 7).

\section{ANALYSE DE LA STABILITÉ}

Une fois les caractéristiques géométriques et géotechniques connues, l'étude de stabilité nécessite le choix d'un modèle de calcul. Les différentes missions de cartographie effectuées par l'IFREMER (sea-beam, plongées de submersibles) en Baie des Anges, et la visualisation des cicatrices de glissements anciens, ont montré que le modèle de glissement à surface de rupture plane permettait une approche réaliste de l'analyse du départ en masse des sédiments. Au choix de ce modèle est associée une condition de calcul, drainée ou non-drainée, selon l'objectif fixé, respectivement à long ou à court terme. L'étude à court terme permet l'adjonction de paramètres dynamiques tels qu'une surpression interstitielle en excès de la pression hydrosta. tique, générée par une vague, la houle ou un séisme, ou encore d'accélérations sismiques horizontales et ver. ticales, elles aussi engendrées par un tremblement de terre. Ces dernières sont exprimées en fraction de l'accélération de la pesanteurg (KARLSRUD and ED. GERS, 1982; ALMAGOR and WISENAM, 1977 et 1982).

Cependant, ces résultats ont montré a posteriori, que l'étude de l'état statique en conditions non-drainées re. venait à simuler un cas très pessimiste qui ne correspondait que partiellement aux observations « de terrain ». En conséquence, il s'avérait nécessaire de formuler des équations de stabilité en conditions drainées, intégrant les paramètres surpression interstitielle et accélérations sismiques, celles-ci ne pouvant être formulées qu'à la condition de considérer les deux derniers paramètres en tant que paramètres statiques (ALMA. GOR and WISENAM, 1977 et 1982 ; KARLSRUD and EDGERS, 1982). Ces équations représentent la possibilité d'apparition d'un phénomène à court terme dans un système à long terme (MULDER et TISOT, 1991). 


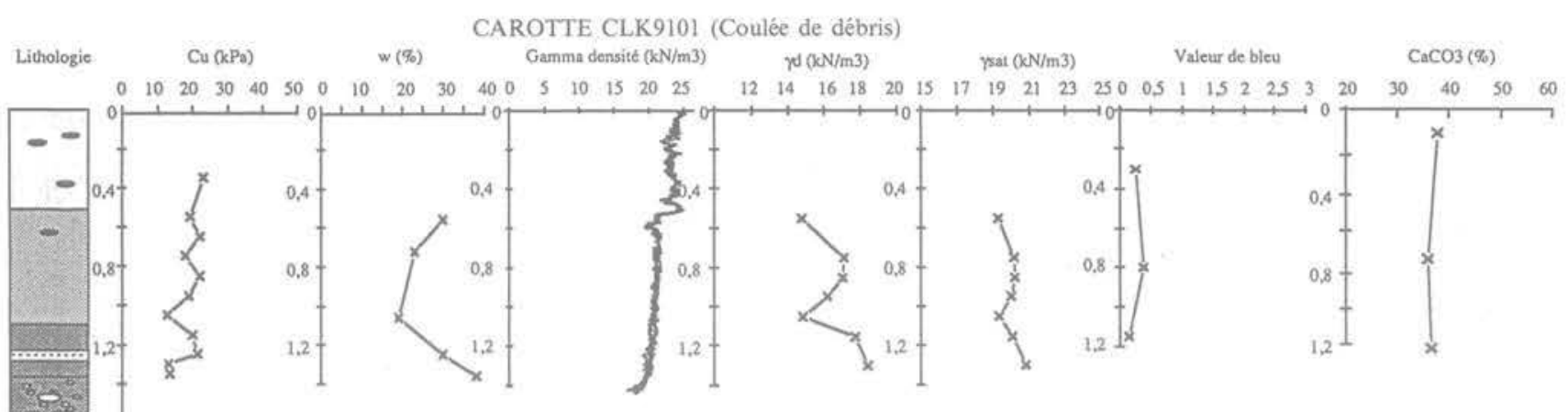

CAROTTE CLK9102 (Silts argileux - rides, plateau continental)

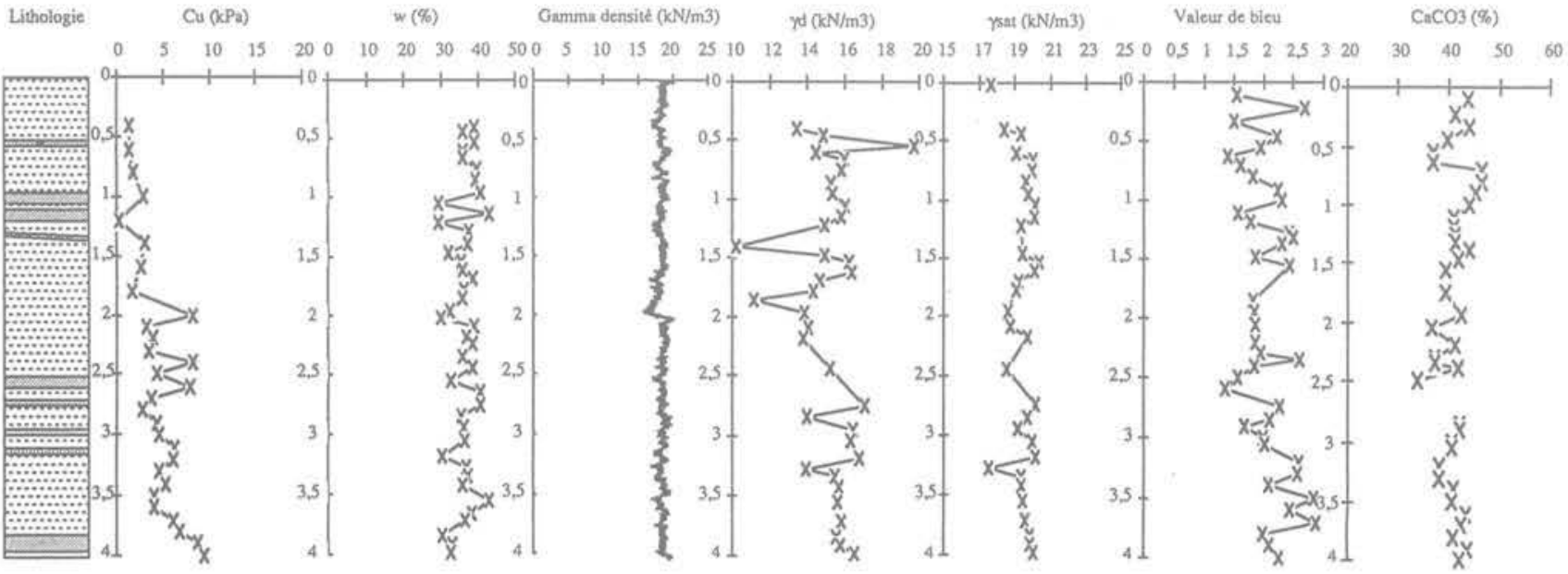

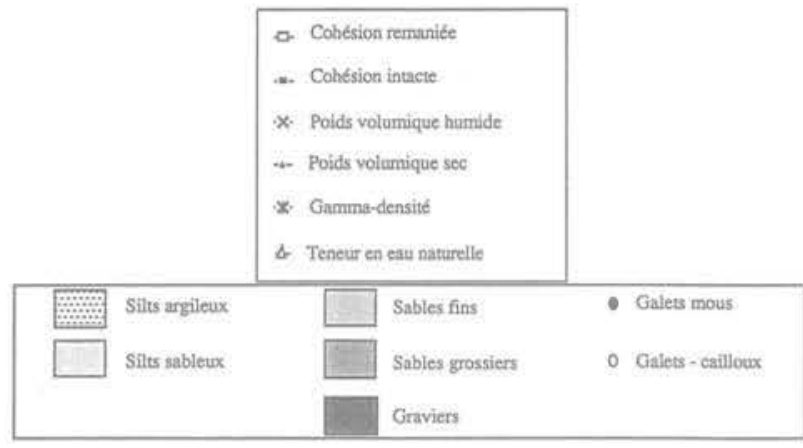

L'utilisation du modèle de glissement plan nécessite le choix de la profondeur de la surface de rupture (z). Ce choix pourrait être effectué à l'aide des résultats de profils sismique réflexion, par mise en évidence d'une surface de discontinuité majeure, tectonique ou, plus probablement, sédimentaire, jouant le rôle de réflecteur. Malheureusement, aucun moyen de sismique réflexion haute définition n'a donné de résultats satisfaisants dans la baie. Parallèlement, peu de carottages Küllenberg dépassent les cinq premiers mètres de sédiments. Les profondeurs de surface de rupture ont alors été définies a priori, en se basant d'une part sur les observations des cicatrices effectuées sur les fonds marins et, d'autre part, sur les calculs de hauteur impliquée dans les glissements anciens à partir des indices de surconsolidation détectées sur carottages et définissant la présence d'un hiatus sédimentaire, avant pour origine un départ en masse de sédiment (COCHONAT and al., 1992; MULDER et al. ; 1992c). De tels calculs ont conduit à une valeur moyenne de $7 \mathrm{~m}$ pour la hauteur glissée. En conséquence, les calculs de stabilité ont été effectués avec des profondeurs de surface de rupture de $5 \mathrm{~m}, 10 \mathrm{~m}$, et $15 \mathrm{~m}$ en conditions drainées, et pour des profondeurs de $1 \mathrm{~m}$ et $2 \mathrm{~m}$, en plus des valeurs précédentes, en conditions non-drainées. La détermination d'une profondeur de surface de rupture fictive revient en fait à estimer pour quelle hauteur critique de sédiment, la rupture $(F=1)$ est atteinte.

Les fonds des canyons du Var et du Paillon n'ont pas été insérés dans la modélisation du fait de leur nature géotechnique très différente. 


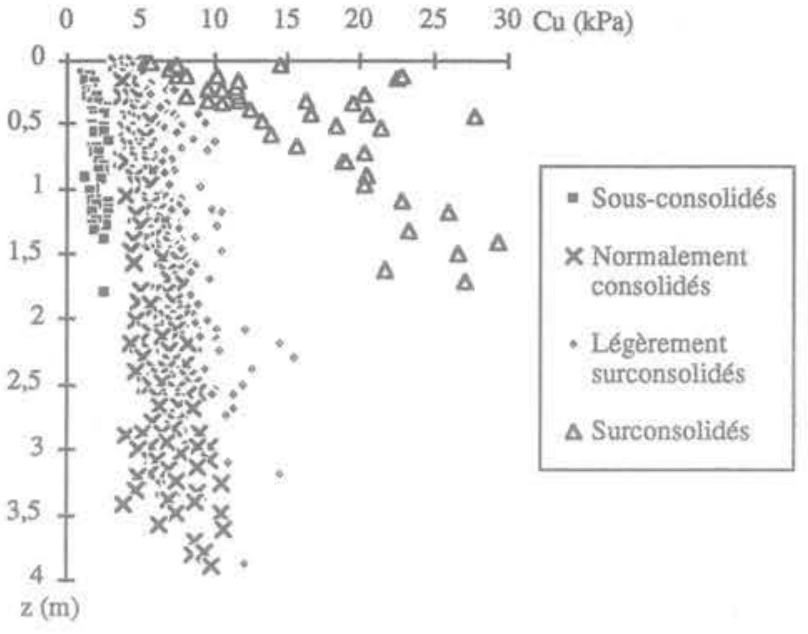

Fig. 6. - Classification géotechnique des sédiments de la Baie des Anges à partir de leur état de consolidation (d'après COCHONAT and al., 1992).

Fig. 6. - Geotechnical classification for the sediments of the Baie des Anges, according to their consolidation state (after COCHONAT and al., 1992).

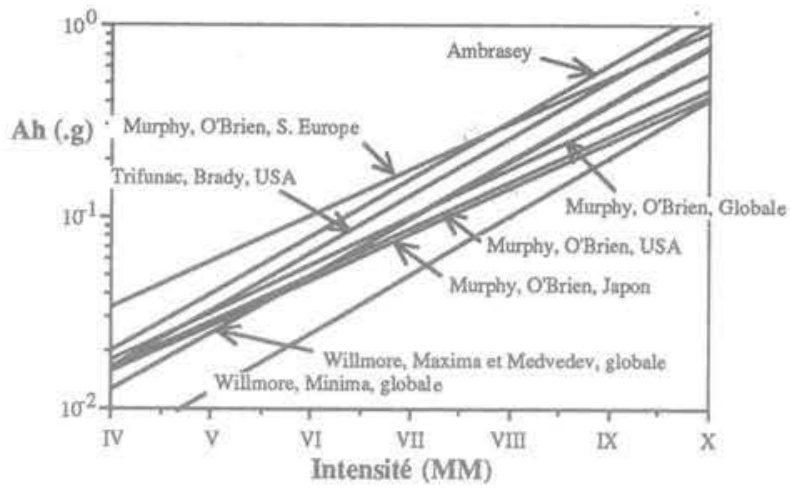

Fig. 8. - Différentes relations empiriques entre le logarithme de l'accélération horizontale (Ah) et l'intensité (Mercalli Modifiée. MM)

(d'après Willmore et Karnik, 1971 ; Despeyroux, 1989).

Fig. 8. - Various empirical relationships between the logarithm of the horizontal acceleration (Ah) and the intensity (MM scale)

(after Willmore and Karnik, 1971 ; Despeyroux, 1989).

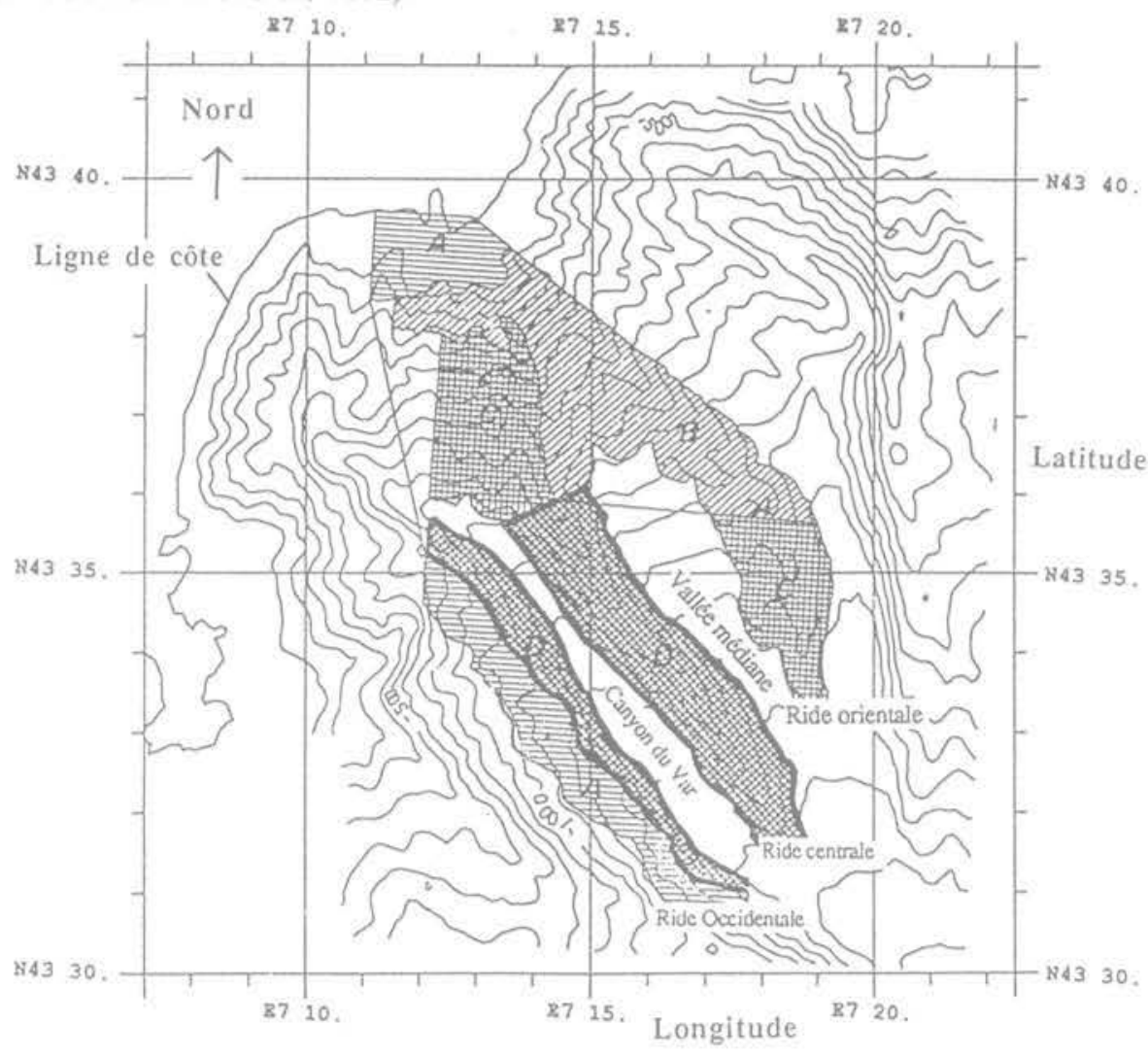

Légende

Famille A (sédiments sous-consolidés)

VIIA Famille B (sédiments normalement consolidés)

Famille $\mathrm{C}$ (sédiments légèrement surconsolidés)

Famille D (sédiments surconsolidés)

Fig. 7. - Carte de répartition des quatre familles géotechniques de sédiment à l'intérieur de la baie (d'après COCHONAT and al., 1992).

Fig. 7. - Map of the distribution of the four geotechnical families of sediments in the Baie des Anges (after COCHONAT and al. 1992). 


\section{ESTIMATION DES PARAMĖTRES EXTERNES}

Les deux paramètres externes principaux intervenant dans le calcul de la stabilité des talus sous-marins sont la surpression interstitielle $(\Delta \mathrm{u})$ et les accélérations sismiques (Ah et Av).

La surpression interstitielle peut être appréhendée de trois manières: la mesure in situ (piézocône), l'estimation à partir d'essais triaxiaux cycliques non-drainés, et enfin, par le calcul théorique à partir d'un échantillonnage de mesures au houlographe (amplitude et période de la houle). De telles mesures ont été fournies par le laboratoire d'Hydraulique de la Direction des Etudes et Recherches d'EDF. Cependant, le calcul de la surpres. sion interstitielle générée par des vagues d'amplitudes annuelles, décennales ou centennales (resp. $3,3 \mathrm{~m}$, $4,2 \mathrm{~m}$ et $5,1 \mathrm{~m})$ a montré que l'impact de $\Delta \mathrm{u}$ sur le coefficient de sécurité était réduit (pour de telles amplitudes, la valeur de $\mathrm{F}$ décroît de 0,1 à 0,2 pour une tranche d'eau de $10 \mathrm{~m}$ ), et que cet impact était toujours masqué par celui de la sismicité. De plus, l'influence de la houle n'est envisageable que pour une tranche d'eau inférieure à $50 \mathrm{~m}$ et n'intéresse donc qu'une surface très réduite de la baie. En conséquence, seule l'influence de la sismicité a été étudiée. On notera cependant qu'en milieu côtier (tranche d'eau inférieure à $50 \mathrm{~m}$ ), l'étude de la houle peut ne plus être négligeable et devra être prise en compte.

Les accélérations sismiques ont été estimées à partir des cartes d'aléa publiées par DADOU et al., (1985). C'est la loi empirique de Murphy et O'Brien (in DESPEY. ROUX, 1990), reliant les accélérations horizontales ou verticales à l'intensité (Mercalli Modifiée, MM), qui a été choisie pour les calculs. Les calculs ont été effectués pour des événements de périodes de retour centennale, bicentennale et millennale.

\section{RÉSULTATS}

La synthèse des résultats de différents cas de modélisation montre d'abord quelques grands traits principaux (MULDER, 1992; MULDER et al., 1992a et 1992b).

Si l'instabilité est plus forte quand on considère une surface de rupture plus profonde, ce phénomène est moins marqué en conditions non-drainées, du fait de lutilisation d'une relation croissante entre $c_{4}$ et la profondeur.

L'étude en conditions non-drainées est, quant à elle, directement liée à la cartographie géotechnique préalable: les zones les plus instables sont celles qui comportent des sédiments sous-consolidés (fig. 9a).

L'étude en conditions drainées en utilisant un critère de Mohr-Coulomb unique pour l'ensemble de la zone fait nettement ressortir l'influence du gradient de pente, au point que la carte de risque, exprimée en isovaleurs du coefficient de sécurité $F$ peut se déduire directement de la carte de gradient de pente (fig. 9b).

Une analyse détaillée montre que les instabilités gravitaires dans la Baie des Anges, peuvent être séparées en deux catégories: celles qui sont liées uniquement aux caractéristiques internes du talus (propriétés géotechniques et géomorphologie) et celles qui apparaissent uniquement sous l'influence de sollicitations externes (sismicité).

Les premières peuvent se diviser en deux grandes familles:

- les instabilités du haut de plateau continental et, dans une moindre mesure, celles qui concernent la partie ouest de la crête occidentale. Elles sont liées à la nature sous-consolidée du sédiment. La profondeur de rupture est située à une profondeur inférieure ou égale à deux mètres. Par conséquent, ces instabilités ne mettent en jeu qu'un volume réduit de sédiment. Etant donné qu'elles sont situées dans une zone préférentielle d'alimentation en sédiment, on peut considérer que ces petits réajustements locaux seront mis en mouvement de façon périodique, dès que la hauteur sédimentée sera égale à la hauteur critique. Leur faible volume et leur situation à un endroit où le gradient de pente prend des valeurs variant de $2^{\circ}$ à $10^{\circ}$ ne leur permettront pas d'effectuer un cheminement important (au plus de l'ordre du kilomètre). Ils se résumeront tout au plus en une translation du matériau, avec possibilité de réaménagement et de formation de coulées de débris; - les instabilités de flanc de crête, affectent les sédiments normalement consolidés, voire surconsolidés. La profondeur de la surface de rupture est située entre 5 et $15 \mathrm{~m}$. Par conséquent, le volume mis en jeu peut être énorme. Situées dans des zones où le gradient de pente prend des valeurs fortes (supérieures à $20^{\circ}$ ), elles pourront acquérir une célérité importante et subir le phénomène d'embrasement (ou d'ignition) pour se transformer en courant de turbidité. Ces courants, caractérisés par un transport en suspension des particules fines, pourront parcourir des distances importantes en suivant la morphologie du fond marin et seront évacués par les canyons du Var et du Paillon, avant de se déposer dans les zones où le faible gradient de pente (de l'ordre du degré) ne permet plus leur avancée.

Ce second type d'instabilités est lié d'une part à l'activité sismique interne de la baie, mais aussi à celle de la mer Ligure (fig. 9c). Une intensité de VII (MM) atteinte à l'intérieur de la baie suffit à déstabiliser les sédiments, en premier lieu ceux situés sur les flancs de la ride centrale, et à générer des glissements de flancs de crête de forte envergure. Un sếisme d'intensité bicentennale entraîne d'importants désordres sédimentaires sur une surface couvrant le quart de la baie (fig. 9d). Un séisme d'intensité millennale, générant une accélération de $0,3 \mathrm{~g}$, engendre une instabilité quasi-générale de la baie.

\section{CONCLUSION}

L'étude des instabilités gravitaires d'origine mécanique en Baie des Anges peut être effectuée grâce au modèle de glissement plan. L'utilisation conjuguée de ce modèle en conditions drainées et non-drainées permet d'effectuer une synthèse réaliste au vu des observations du fond marin, c'est-à-dire en tenant compte des caractéristiques des glissements anciens. Les résultats bruts présentés sur la carte de risque (fig. 10) sont cependant à modérer. Il est clair par exemple, qu'un événement tel qu'un séisme d'intensité millennale n'est pas à considérer comme un accident ponctuel dans un système 


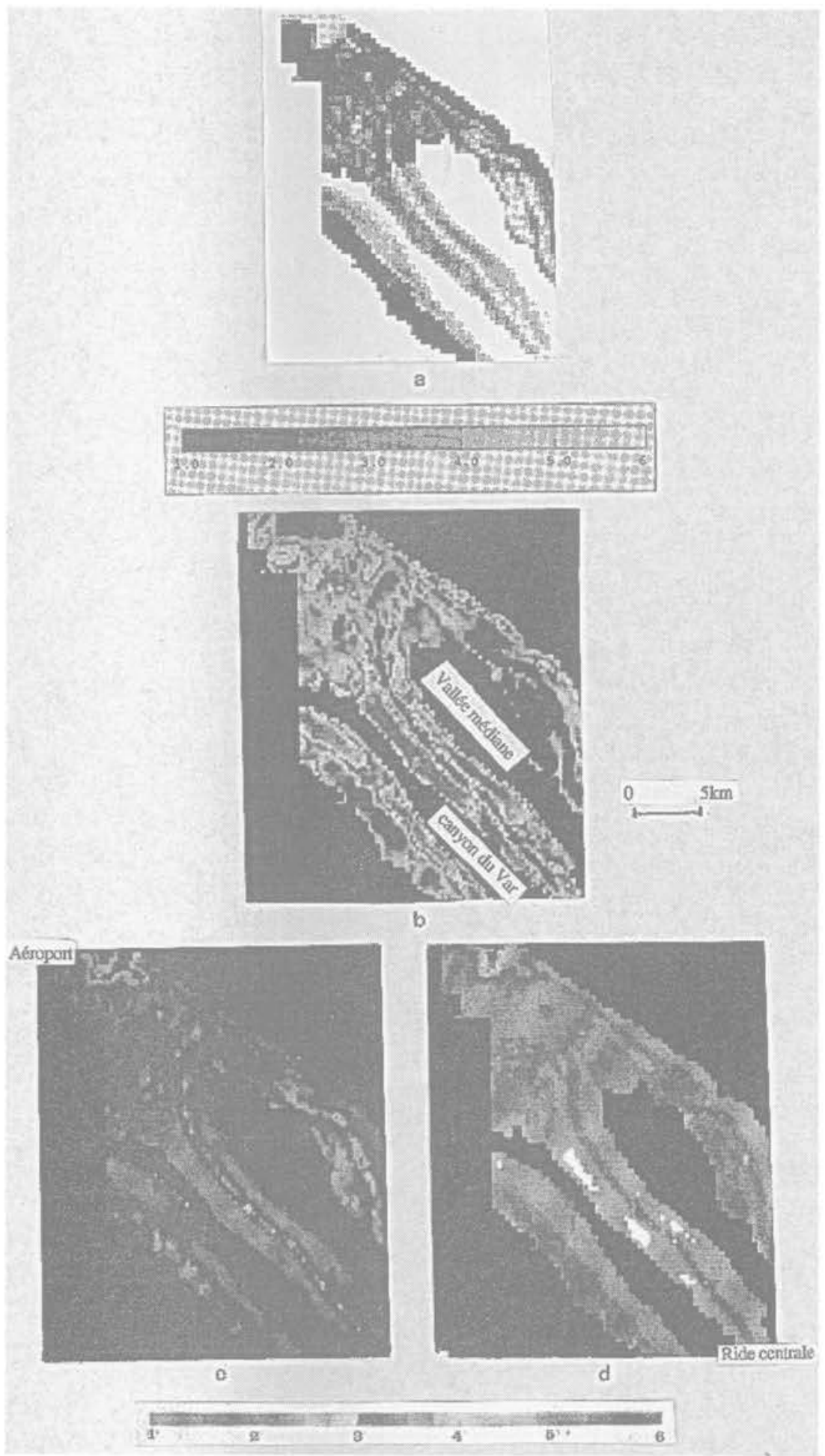

Fig. 9. - Différents cas de simulation des instabilités

$a$ : conditions non-drainées, $z=2 \mathrm{~m} ; b$; conditions drainées, $z=10 \mathrm{~m}$; $c$ : conditions drainées, influence d'une accélération centennale, $z=10 \mathrm{~m}$ d: conditions drainées, influence d'une accélération bicentennale, $z=10 \mathrm{~m}$. (Les zones ayant une valeur du coefficient de sécurité égale à 1 sont en blanc). Fig. 9. - Cases of instabilities simulations:

a: undrained conditions, $z=2 \mathrm{~m}: b$ : drained conditions, $z=10 \mathrm{~m}$ : c: drained conditions d, influence d'une accélération centennale, $z=10 \mathrm{~m}$. $d$ : drained conditions, influence d'une accélération bicentennale, $z=10 \mathrm{~m}$. (Areas with a security factor value equal to 1 are in white). 


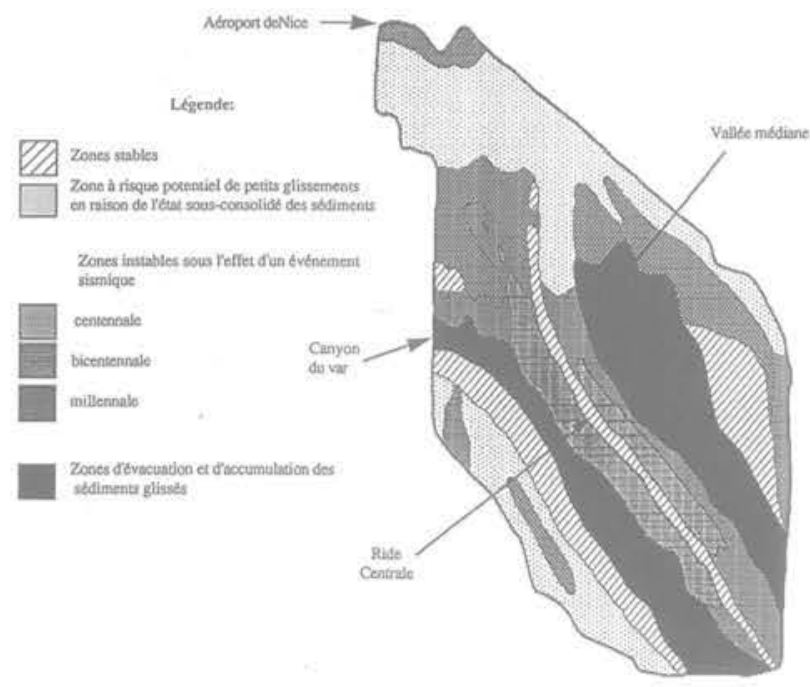

Fig. 10. - Carte synthétique du risque d'instabilité en Baie des Anges.

Fig. 10. - Synthetic map of hazards in the Baie des Anges.

statique. La baie constitue un système dans lequel les sédiments sont soumis à un mouvement continu de dépôt et de départ ou de déplacement. Dans la baie, il semble que les mouvements de sédiments postérieurs à leur dépôt seront essentiellement des glissements en masse. Un séisme de période de retour égale à 1000 ans se produira alors dans un système à l'inté. rieur duquel une masse importante de sédiments aura été « purgée » par des événements de période de retour moindre ou encore par des glissements gravitaires simples n'ayant pas été engendrés par un événement extérieur.

Les zones cartographiées comme «zones à risques * doivent, en tenant compte des précautions précédentes, être considérées comme des zones à risque potentiel, sous réserve que les hypothèses formulées avant le calcul soient effectivement celles qui régissent le système au moment de l'événement. Dans ce cadre, les zones potentiellement instables sous l'effet d'un séisme d'intensité millennale seront celles présentées sur la figure 10 à l'exception de celles qui ne vérifieront pas la principale hypothèse de calcul, à savoir la profondeur de la surface de rupture, soit qu'elles auront été préalablement "purgées », soit qu'une variation locale ou temporelle du taux de sédimentation fera en sorte que la hauteur sédimentée au-dessus d'une surface de rupture potentielle sera moindre que la hauteur critique entraînant la rupture. Dans ces conditions, il ne faut donc pas considérer qu'un séisme de période de retour millennale engendrera le départ d'une masse unique de sédiment de plusieurs dizaines de millions de $\mathrm{m}^{3}$, ordre de grandeur qui est contredit par les observations, mais générera plutôt tout un ensemble de petits glissements plans de volume moindre (quelques millions de $\mathrm{m}^{3}$ au maximum), successifs ou simultanés. Dans le second cas, les glissements pourront alors devenir coalescents au niveau des canyons du Var et du Paillon pour former un courant de turbidité de taille importante. Dans ce cadre, il semble qu'un séisme d'intensité supérieure à VII (MM) serait susceptible d'entraîner des désordres sédimentaires importants dans la baie. Là encore, une synthèse des résultats des calculs menés à la fois en conditions non-drainées et en conditions drainées, en considérant les accélérations sismiques en tant que paramètres statiques, permet une estimation réaliste de ces désordres. Cette démarche permet de plus, la mise en évidence du type d'instabilité qui sera prépondérant dans une zone donnée, quelle en sera son origine, sousconsolidation du sédiment superficiel, fortes valeurs du gradient de pente ou sismicité, et par conséquent, quel sera son évolution, désordre local, ou transition vers un courant de turbidité.

Il est difficile de proposer une méthode de prévention spécifique contre le risque de glissement dans la baie. A l'échelle régionale, aucune méthode globale de prévention r'est applicable. Néanmoins, à l'échelle non plus de la carte, mais du plan, certaines possibilités peuvent être soumises. En haute mer, la seule alternative est une * purge * locale des sédiments superficiels, qui aurait l'avantage de réajuster le gradient de pente par élimination de la tranche de sédiments sous-consolidés, c'est-à-dire de se prémunir contre les deux causes principales de glissement. Cependant, si cette méthode semble être, en théorie, la seule efficace, il faut considérer qu'une telle action se place dans un milieu hostile à tout aménagement géotechnique classique. Si la technique de purge est utilisée de façon routinière en milieu continental (tranchées, déclenchement d'avalanches), les outils de haute technicité à mettre en œuvre et la difficulté d'accès en milieu marin font que de telles méthodes sont difficilement applicables à l'heure actuelle. Il faut de plus considérer qu'une telle purge en mer ne pourrait s'effectuer qu'à l'explosif dont l'utilisation pourrait avoir des conséquences difficilement mesurables a priori, par exemple, la génération d'accélération du sol (vibrations), d'une surpression interstitielle ou encore l'impact causé à l'environnement.

Néanmoins, cette étude montre, qu'à l'instar du delta du Mississippi, on peut concevoir la Baie des Anges comme un cas d'école dans l'étude des instabilités gravitaires sous-marines d'origine mécanique. Leur risque d'apparition doit engendrer une étude approfondie lors des travaux futurs d'aménagement de la baie.

\section{BIBLIOGRAPHIE}

ALMAGOR G. and WISEMAN G.H. (1977), Analysis of submarine slumping in the continental slope of the southern coast of Israël. Marine Geotech., Vol. 2, Marine Slope Stability, pp. 349-380.

ALMAGOR G. and WISEMAN G.H. (1982), Submarine slumping in the continental slope of Israël. Marine Slides and other mass movements. Proceeding of a NATO Workshop on marine slides and other mass movements, Algarve, Portugal, 15-21 dec. 1980, Sér. 4, Vol, 6, S. Saxov et J.K. Nieuwenhuis ed., pp. $95 \cdot 128$.

AUFFRET G.A., AUZENDE J.M., GENNESSAUX M., MONTI S., PASTOURET L., PAUTOT G. and VANNEY J.R. (1982), Recent mass wasting processes on the provencal margin (Western Mediterranean). Marine Slides and other mass movements. Proc. of a NATO Workshop on marine slides and other mass movements, Algarve, Portugal, $15-21 \mathrm{dec} 1980$, Sér. 4, Vol. 6, S. Saxov et J.K. Nieuwenhuis ed., pp. 53-58.

BOURILLET J.F. (1991), Géomorphologie à partir d'un modèle numérique de terrain (Baie des Anges, Nice), $3^{e}$ congrès français de Sédimentologie, Brest. 
CLARK J.I. and LANDVA J. (1991), Geotechnical aspects of seabed pits in the Grand Banks area. Can. Geotech. J. 25 , pp. $448-454$.

COCHONAT P., SCHIEB T., GUILLAUMEJ., KERBRAT R. TISOT J.P. AUFFRET G.A and MULLER C. (1988), Geotechnical and sedimentological properties of Nice slope and submarine Var canyon deposits. Mediterranean Basins Abstracts, AAPG. convention, feb. 1988.

COCHONAT P., SCHIEB T., MULIER C., BOURIL. LET J.F. et SAVOYE B. (1989), Hiatus sédimentaires et surconsolidation. Indices de glissements superficiels dans le cône sédimentaire sous-marin du Var. $2^{e}$ congrès de Sédimentologie, Paris, 20-22 nov. 1989, pp. 77-78.

COCHONAT P. DODD L., BOURILLET J.F. and SA. VOYE B. (1992), Geotechnical characteristics and instability of submarine slope sediments, the Nice slope ( $N$-W Mediterranean sea), a case study. Marine Geotechnology, sous presse.

COCHONAT P. DAMYG.. LEDUC B., MEUNIER J. HARMEGNIES F. et FLOURY L. (1990), Mesures géotechniques par grand fond. Coll. ISM, Toulon, 3-5 dec. 1990.

COCHONAT P. (1991a), High resolution geophysical investigations of submarine slope. Intensive course, 19. 31 aug. 1991, Patras.

COCHONAT P. (1991b), Deep in situ geotechnical mea surements. Intensive course, $19-31$ aug. 1991, $\mathrm{Pa}$ tras.

COLEMAN J.-M. and GARRISON L.E. (1977), Geological aspects of marine slope stability, Northwestern gulf of Mexico. Marine Geotech., Vol. 2, Marine Slope Stability, pp. 9-44.

COLEMAN J.M. and PRIOR D.B. (1981), Subaqueous sediment instabilities in the offshore Mississippi river delta. Offshore Geologic Hazards, Short courses of Offshore Tech. Conf., section 5, univ. Rice, 2-3 mai 1981.

DADOU $C$. GODEFROY $P$. et VAGNERON J.M. (1984), Evaluation probabiliste de l'aléa sismique ré. gional dans le Sud-Est de la France. Docurnent du BRGM, n 59, 246 p.

DESPEYROUX J. (1989), Construction en zone sismique, Mémoire concernant les niveaux d'agression à considérer pour l'application des recommandations de l'AFPS relatives aux constructions à édifier dans les régions sujettes à séismes. Cahier spécial de l'AFPS, $N^{\circ}$ spécial «Accélérations nominales », 48 p.

GENNESSAUX M., MAUFFRET A. PAUTOT G. (1980), Les glissements sous-marins de la pente continentale niçoise et la rupture de câbles en mer Ligure (Méditerranée occidentale). C.R. Acad. Sc., t. 290, pp. 959-962.

HEEZEN B.C. and EWING M. (1952), Turbidity currents and submarine slumps, and the 1929 grand banks earthquake. Am. J. of Sc., Vol. 250, pp. 849-873.

KARLSRUDK. and EDGERS L. (1982), Some aspects of submarine slope stability. Marine Slides and other mass movements. Proc. of a NATO Workshop on marine slides and other mass movements. Algarve, Portugal, 15-21 dec. 1980, Sér. 4, Vol. 6, S. Saxov et J.K. Nieuwenhuis ed., pp. 63-82.

KUENEN P.H. (1952), Estimated size of the grand banks turbidity currents. Am. J. of Sc., Vol. 250, pp. 874 884.
MULDER Th. et TISOT J.P. (1991), Méthode générale d'étude de l'instabilité des sédiments superficiels en milieu marin. $3^{\mathrm{e}}$ congrès français de Sédimentologie, Brest.

MULDER Th. (1992), Approches géotechniques de la sta. bilité des marges continentales; application à la Baie des Anges, Nice, France. Thèse INPL-ENSG.

MULDER Th., TISOT J.P., COCHONAT P. et BOURILLET J.-F. (1992a), Mêthode d'analuse des instabilités en mer. Exemple de la Baie des Anges, Nice, France. $14^{\mathrm{e}} \mathrm{RST}, 14.17$ avril 1992 . Toulouse.

MULDER Th. and TISOT J.P., COCHONAT P. et BOU. RILLET J.F. (1992b), Instability of superficial sediments in «Baie des Anges * (Nice, France). Actes du colloque franco-italien "Slope stability in seismic Areas", 14-15 mai 1992, Bordighera, Italie, E. Faccioli and A. Pecker eds, pp. 125.138.

MULDER Th. COCHONAT P., TISOT J.P. et BOURIL. LET J.F. (1992c), Détermination de la hauteur des glissements anciens à partir de l'état de consolidation des sédiments en Baie des Anges (SE de la France), C.R. Acad. Sci, Paris, t. 315, série II, pp. 1703-1709.

PRIORD.B. and COLEMAN J.M. (1982), Active slides and flows in underconsolidated marine sediments on the slope of Mississippi delta. Marine Slides and other mass movements. Proc. of a NATO Workshop on marine slides and other mass movements, Al. garve, Portugal, 15-21 dec. 1980, Sér. 4, vol. 6, S. Saxov et J.K. Nieuwenhuis ed., pp. 21-50.

REHAULT J.P. et BETHOUXN. (1984), Earthquake relocation in the ligurian sea (western Mediterranean): geological interpretation. Marine Geol., 55, pp. 429. 445.

SAVOYE B., COCHONAT P., OLLIER G., AUFFRET G.A. and BOURILLET J.F. (1989), The submarine Var canyon (french riviera): detailed study of the activity of a modern canyon. 10th regional meeting of sedimentology, Budapest (Hungary), april $24 \cdot 26,1989$

SAVOYEB. and PIPER D.J.W. (1991). The Messinian event on the margin of the Mediterranean sea in the Nice area, southern France. Marine Geol., 97. pp. 279.304.

SCHIEB Th. TISOT J-P et COCHONAT P. (1990), Propriétés géctechniques et étude de la stabilité des sédiments marins de la Baie des Anges (Nice, AlpesMaritimes). ASF, $2^{e}$ congrès Fr. de Sédimento, Paris.

SCHIEB Th. (1992), Faciès géotechniques et état de consolidation des sédiments de la pente continentale nicoise (Baie des Anges). Thèse doct. INPL Nancy. $190 \mathrm{p}$.

SKEMPTONA.W and HUTCHINSON J.N. (1969), Stability of natural slopes and embankment foundations. Proc of 7th conf on Soil Mechanics and Founda. tion Engineering. State-of-the-art, pp. 291-340. Mexico.

TISOT J.P. (1986), Propriétés géomécaniques des sédi. ments des grands fonds océaniques. Thèse d'Etat, ENSG-INPL, Nancy, coll. mémoires des Sciences de la Terre, $n^{\circ} 46$, éd. Fondation Scientifique de la Géologie et de ses Applications.

TISOT J.P. et MULDER Th. (1990), Méthodes d'études de la stabilité des talus sous-marins. $3^{e}$ congrès franco-polonais, Wroclav, Pologne, $15-16$ mars 1990.

WILLMORE P.L. et KARNIK K.-V. (1971), Manuel pratique des observatoires sismologiques. Centre sismologique international, Edimbourg. 